\title{
Introduction to Sociocybernetics (Part 3): Fourth Order Cybernetics
}

\author{
Roberto Gustavo Mancilla \\ LLB. Instituto Tecnológico y de Estudios Superiores de Monterrey, \\ JSD, LLM University of California, Berkeley \\ robi357@hotmail.com
}

Table of Content:

Introduction

1. Fourth order cybernetics

a) Points of contact between second and fourth order cybernetics

b) Fourth order cybernetics as the realm of self observing systems

c) Cognitive machines

d) Rationality as a cognitive machine

e) Heuristical aspects of Cybernetics: Order, balance and harmony as measures of the observer.

i Definitions

ii Order, Balance and Harmony in Rationality

iii Constructivist epistemology. A brief introduction

iv Social morphogenesis

2. The relationship between third and fourth order cybernetics

a) Rationality and Language

b) Application of fourth order cybernetics to Society, Power, Culture and Institutions

c) Sociocybernetics

Conclusion 


\section{Introduction}

The purpose of this paper is to complement those that have preceded, changing the focus from the dynamics of social systems to that of individual human systems. It will be seen that fourth order cybernetic systems study self-observing systems, which are comprised by cognitive machines, information processing mechanisms that reside in the human mind. Of these, the one of rationality will be explored and then related to that of language, which acts as cognitive bridge between human systems.

The idea of rationality as a cognitive machine that has as its purpose though coherence will be offered to the reader as the staple of fourth order cybernetics; another aspect of it will be that of heuristics, not only as practical reasoning but as ways of conceiving and understanding the world. The framework that will be offered will consist of the understanding of patterns (Order), their proportion (Balance) and Harmony as their functional conjunction; constructive epistemology will also be delved upon in order to create a complete perspective upon human psychic systems.

Finally, the ideas of fourth order cybernetics will culminate in the idea of social and cognitive morphogenesis as heuristics is related to measures of complexity: order will be related to hierarchy, balance to self-similarity and harmony to universality; it will be concluded that repetition is the most adequate measure of complexity in social systems.

An explanation of the relationship between rationality and language will also be offered and a brief relation between Society, Power, Culture, Institutions and Rationality will be made and finally, the concept of sociocybernetics will be approached in light of the idea of third and fourth order cybernetics, with a redefinition of it being advanced.

\section{Fourth order cybernetics}

\section{a) Points of contact between second and fourth order cybernetics}

Self consciousness is the point of transition between lower cognition (which pertains second cybernetics) and that which belongs to human beings (which is the object of study of what will be called fourth order cybernetics), which has been called high cognition in the previous parts of this study. It was said before that this was so because of the self-consciousness that a system can acquire with self observation, and thus be teleonomical and teleological. Before entering the study of fourth order cybernetics, it is necessary to further develop the notion of cognition, so the analysis will be complete. 
In his widely known psychological research, Piaget makes a typology of the cognitive development of a human being since its birth to adulthood: ${ }^{1}$

- Sensorimotor stage (from birth to a year and half, two): The first motor reflexes develop, along with first instincts and emotions, there is also a development of a sensatory-motor intelligence previous to language. Knowledge starts developing on the basis of experiences/interactions; some language skills are developed at the end of this stage.

- Pre-operational stage (from two to seven years): Language skills are present, intuitive intelligence develops, there is a submission to adults and spontaneous intersubjective feelings; memory and imagination are developed.

- Concrete operational stage (from seven to twelve years): Logical and systematic intelligence blossoms, along with moral and social sentiments of cooperation; manipulation of symbols related to concrete objects; operational thinking predominates.

- Formal operational stage (adolescence to adulthood): Abstact intellectual operations appear, personality forms and there is an affective and intellectual insertion into adult society.

In every state is distinguished from its preceding one because of the appereance of new original cognitive structures. In this typology, the difference between lower and higher cognition can be seen more clearly: While in the sensorimotor there is motor activity, knowledge based on experience and interaction and limited language acquisition, in the formal operational stage an individual can communicate to others by means of a symbol system and capable of logical and abstract reasoning.

This is the transition between the lower cognition in animals and primates who have it along a limited degree of self observation, and human beings, which are capable of higher cognition by means of language, abstraction and formal reasoning.

Higher cognition was defined priory, but a reprisal of the concept is useful: It is the processing (storage, retrieval, transformation, creation and transmission) ${ }^{2}$ of information made by an autopoietic system in its interaction with what surrounds it (environment and other beings) with the possibility of stating a purpose beyond selfsustainment.

\footnotetext{
${ }^{1}$ Piaget J., Petit N. (trans.) (1974) Seis Estudios de Psicologia[Six Studies in Psychology], Editorial Seix Barral, 14 and 15.

${ }^{2}$ Stillings N.A., Weisler S.E., Chase C., Feinstein M.H., Garfield J.L., Rissland E.L. (1995) Cognitive Science. An Introduction, (2nd ed.), MIT Press, 1.
} 


\section{b) Fourth order cybernetics as the realm of self observing systems}

Fourth order cybernetics deals with the study of self observing systems, which are both teleological and teleonomical; because of this, fourth order systems (and third order too) have features of both first and second order cybernetic machines. W. Ross Ashby (1965), one of the most notable developers of cybernetics, in his famous introductory work to this science, states that it is a "theory of machines", which focuses not on the things studied, but on their behavior. His point can be elaborated in the following quote: ${ }^{3}$

It takes as its subject-matter the domain of "all possible machines", not yet been made, either by Man or Nature. What cybernetics offers is the framework on which all individual machines may be ordered, related and understood.

Fourth order cybernetics studies cognitive machines, information processing mechanisms of the high order that have their basis within the neural network of human beings, that is, it is the cybernetics of human beings. There are many cognitive machines that make up higher cognition, however, the one to be preeminently studied by this branch of cybernetics is rationality, understood as a mechanism which allows the development of coherence within the thought system and also its relationship to language, understood as the cognitive machine that complements rationality and also the one that allows the bridging of cognitive systems, thus fostering socialization.

Ashby's (1965) definition of cybernetics as the domain of "all possible machines" gives way to the creation of a fourth (and third) order in cybernetics that studies the cognitive mechanisms of the human brain, Stillings et al.(1995) harmonize with this latter idea when they state that:

The high degree of flexibility of human cognition requires that we think of much of the human cognitive architecture not as determining specific thoughts and behaviors but as an abstract set of mechanisms that potentiates a vast range of capabilities.

Finally, to close this topic, it is necessary to review other attempts at creating a notion of fourth order cybernetics. Fortunately for the author, the entrenchment of the students of second order cybernetics and their insistence that the latter is enough to analyze human phenomena (as in high cognition and social systems) has restricted the interest in the crafting of a third, lest a fourth order of cybernetics.

The only attempt made to create a fourth order of cybernetics by the time this study was created was made by Zangeneh and Haydon, which create a cybernetic model of the human psyche (a psycho-structural model by their own admission) to address

\footnotetext{
${ }^{3}$ Ashby W. R. (1965) Introduction to Cybernetics, Chapman Hall Ltd., 1.
} 
problem gambling in a six page paper of which, the model of cybernetics is not to be found. After this brief paper there is no other attempt to further define cybernetics, although a manuscript submission for publication is alluded. ${ }^{4}$ Due to the scarcity of the material, a selective transcription of the authors is better fitted than a description. As purposes for the development of a new version of cybernetics, they stated:

- To introduce a new paradigm shift.

- To address an explicit discussion of the human system, including problem gambling.

- To develop a model to demonstrate how structure and context influence such systems

On the scope of their model they said:

Psycho structural cybernetics is a model derived from the fourth order cybernetic theory. In this model, the basic units of a human system are the agent and the structure... These two components provide feedback to one another and influence one another's activities. Within the agent component, both biological and psychological factors have an impact. Information and activities that are contained within the agent have the activity to interact with the structure through the more general agent structure loop. The structural component includes elements such as culture, broadcast agents, economical disparity, political and public health policy, and community structure...In the basic feature of the human system, feedback occurs between two integral components (agent and structure).

Although there is resonance between Zangeneh and Haydon's (2004) notion of fourth order cybernetics and the one advanced in this study, the main difference between them reside in the approach, for the former adopts a psychological and postmodern approach that assimilate cybernetics into this discipline and school of thought respectively, while the latter attempts not an interdisciplinary approach, but a transdisciplinary one, that is, there is an attempt to develop a model that does not fit within the boundaries of a specific branch of the social sciences, but one that respects the basic tenets of cybernetics.

\footnotetext{
${ }^{4}$ Zangeneh M., Haydon E. (2004), The Psycho-Structural Cybernetic Model, Feedback, and Problem Gambling: A New Theoretical Approach, InTERnATIONAL Journal OF MENTAL HeALth AND AdDiction, Vol. 1, No. 2, pp. 25-31.
} 


\section{c) Cognitive machines}

Two questions are to be asked in understanding cognitive machines: First, what are the elements that constitute such mechanisms? Second, what are the defining features of a cognitive system? As it was said before, three are the requisites to be fulfilled for a machine to be considered as cognitive:

- It must store and retrieve information.

- It must help to understand received information.

- It must create new information.

The defining features of cognitive machines can be expounded by analyzing their relation to their inputs and outputs; cognitive machines receive, create, transform and transmit information, which is both their input and output, and it can be used either to create new data, different from that received or to broaden the existing information storage in the brain, which can result in the expansion of the cognitive domain.

Again, cognitive machines are omnipoietic because they can produce both their own components and information other than itself; omnipoiesis, the ability to create all kinds of output (internal and external to self) is the distinguishing features of cognitive machines, which are the subject of study of third and fourth order cybernetic systems.

\section{d) Rationality as a cognitive machine}

Rationality is a cognitive machine that has as a purpose of maintaining the coherence of a cognitive system, such is the object of study of fourth cybernetics. What are the meaning, scope and purpose of thought coherence?

There are a myriad of criteria regarding the content of rationality. For the purpose of this study, rationality will be understood as the human capacity of making sense of things and retaining the coherence of its cognitive domain. It is a cognitive machine because it involves storage and retrieval of information and the assimilation of and creation of new information, that is, it is an information processing function of the brain.

The purpose of rationality is to serve as an adaptation mechanism that helps the cognitive domain to deal with past and current experience, arranging, rearranging existing information according to a vision of the world, or rearranging that cosmovision in the light of inconsistencies. Rationality in the fourth cybernetic sense is a coping mechanism. 
An important feature of rationality and possibly of other cognitive machines is what can be called reflexivity, which is not the feature of coherence, but rather what allows its existence. Reflexivity is the process of self-observation and deliberation that results in rational behavior; as such, it is a staple of fourth order cybernetics and of rationality as a cognitive machine. It is important to note that rationality as thought coherence is not fully bound to the human mind, that is, because rationality is information and as such can be transmitted, as knowledge that it is, it can also be constructed by groups or by bigger social systems.

Rationality can have three dimensions: an individual in which a human sets his own criteria on what constitute coherent thoughts and behavior, a groupal (there can be several and its nature vary according to the type of association), in which a specific set of individuals create a consensus on what is coherent behavior and a social one in which, this consensus is mediated on a much larger scale (as in a State, confederation of States or a world system).

It is important to realize that the dimensions of rationality do not flow smoothly all the time. The individual level of rationality can be at odds with a groupal or a social one, thus creating a cognitive dissonance, that is, what one level considers rational is conflicted with the standards of another level.

In such cases, there can be three scenarios in which the rationality of a system would attempt to smooth the incongruencies:

- One level of rationality might attempt to influence the other in order to diminish or eliminate the conflict, for example, an individual attempting to influence a group or society in general.

- Individual rationality as a coping system would attempt to reconciliate incongruencies in order to safeguard the overall coherence of the thought system.

- One level of rationality would attempt to ignore such incongruence in order to avoid the conflict.

It has to be noted though that rationality, both individual and collective, in some measure depends on language and the language also relies on thought coherence to develop. Cognitive machines are deeply related one to the other, though they hold certain degree of autonomy and sometimes tension can arise between two or more cognitive machines. The full relationship between rationality and language will be ascertained further down this study. 


\section{e) Heuristical aspects of Cybernetics: Order, balance and harmony as measures of the observer.}

\section{i) Definitions}

Heuristics commonly refers to refers to practical techniques for problem solving, learning, and discovery; as part of fourth order cybernetics, heuristics will be understood as the basic understanding for perceiving and interacting with the environment, which is done in terms of order, balance and harmony.

The Oxford Dictionary defines order as "the arrangement or disposition of people of things in relation to each other according to a particular, sequence, pattern or method" 5 and pattern as "regular and intelligible form or sequence discernible in the way in which something happens or is done". ${ }^{6}$ W.D. Oliver (1951) defines it as "an arrangement of a set of entities that is produced by the correlation, according to a rule, of one arrangement of these entities with another arrangement independent of the first". ${ }^{7}$

Both definitions have the following elements:

- Ordered entities (people or things)

- A rule or pattern under which the objects are arranged

- A relationship between the ordered objects.

George Nakhnikian (1954) criticizes the generality of Oliver's idea of order by questioning its applicability to quantum theory, where the features of the entity (selfidentity and distinguishability) are operationally meaningless and he proposes a notion of order as "whatever is described by law". 8

This counter-definition might have problems in being applied to moral and legal norms, which unlike scientific laws that describe phenomena, have content that might be violated or changed by its subject; this being said, Nakhnikian's idea of order needs to be refined from whatever is described by law to whatever is described by men by means of a law. Both notions of order share the following feature: A secondary arrangement, that is, the existence of an orderer, who establishes the first arrangement.

\footnotetext{
5 Order Definition in Oxford Dictionary:

http://oxforddictionaries.com/definition/order?region=us\&rskey=roqJp6\&result=1

${ }^{6}$ Pattern Definition in Oxford Dictionary:

http://oxforddictionaries.com/definition/pattern?region=us\&q=pattern

${ }^{7}$ Oliver W. D. (1951) Theory of Order, The Antioch Press, 19.

8 Nakhnikian G. (1954), A Note on W. Donald Oliver's Theory of Order, PHILOSOPHY OF SCIENCE vol. 21, No. 2, pp. 169-172.
} 
The idea of order does not exist outside of human beings; in Searle's terminology it is an institutional fact, not a brute one. Why this is so? It is because scientific criteria and other social criteria notwithstanding, what to one person constitutes as order might appear to another as disorderly; it is a construction of the human mind. Order is relative to the mind of the observer.

In this tenor of things, it might be said then that the dichotomy between of order and disorder is inexact because it is not total ${ }^{9}$, therefore, one might instead talk about different types of order. Again, order is subjective in nature, created by the mind of the individual taking into account factors like social context, life experience, training and others, but there can be consensus among individuals regarding acceptable types of order: this goes from scientific laws describing phenomena to social norms of behavior.

Garcia Maynez (1974) applies Oliver's idea to Law, but first gives his initial interpretation of the latter: "Order is the submission of a set of objects to a rule or a system of rules which application creates among said objects, the relationships that allow the goals of the orderer". ${ }^{10}$ This definition presupposes all of the aspects seen in previous paragraphs: A set of objects (which the author later expands to include people), an ordering pattern, the submission of the former to the latter, the relations that derive from such submission to the ordered objects, the goal pursued by the orderer. Another presupposition that can be added to this list is the relationship that the ordered have with the orderer and the pattern.

In Garcia Maynez's transition from a general notion of order to that of a normative order, he states several principles that are useful to understand how order is applied in a social context:

- The notion of "objects" ("entities" in the case of Oliver) are not limited to physical objects, they can also comprise human conduct that goes beyond their own thoughts.

- The nature of the order depends of the type of rules used: scientific rules which describe a phenomenon (something that "is") differ from rules of behavior conduct that seek to establish a specific conduct (what "ought to be").

- The rule is also conditioned by the nature of the objects, which limit the possibility of ordering and also by the goals pursued by the orderer.

- Orders established by men always tend to a purpose, that is, they are instrumental in nature; there is no ordering for its own sake.

\footnotetext{
${ }^{9}$ It does not comprise precise criteria to the totality of human beings.

${ }^{10}$ Máynez E.G. (1974) Filosofía del Derecho[Legal Philosophy], Porrúa, 23.
} 


\section{Roberto Gustavo Mancilla}

He also distinguishes between the "conception" and the "effective accomplishment" of an order, and says that there are 3 stages:

1. Conceiving the order.

2. Electing the means to achieve the goal.

3. The effective accomplishment of the projected order.

Balance is understood by the dictionary as "a condition in which different elements are equal or in the correct proportions". ${ }^{11}$ Proportion is also defined as: a part, share, or number considered in comparative relation to a whole". This idea is used more in the arts than in the sciences, but like the notion of order, it is relative to the human observer and extends to his behavior.

In design, balance is a principle that seeks the reconciliation of opposing forces in a composition that results in visual stability. It can be classified into: „symmetrical“ and ,asymmetrical“. ${ }^{12}$

„Symmetrical balance“ is the equal distribution between the sides of a centrally placed fulcrum. When the elements are arranged equally on either side of a central axis, the result is „,bilateral“ symmetry, which can be horizontal or vertical. Another type of symmetry occurs by arranging elements equally around a central point, this is known as „radial“ symmetry. On the other hand, asymmetrical balance is the placement of objects in a way that will allow objects of varying visual weight to balance one another around a fulcrum point.

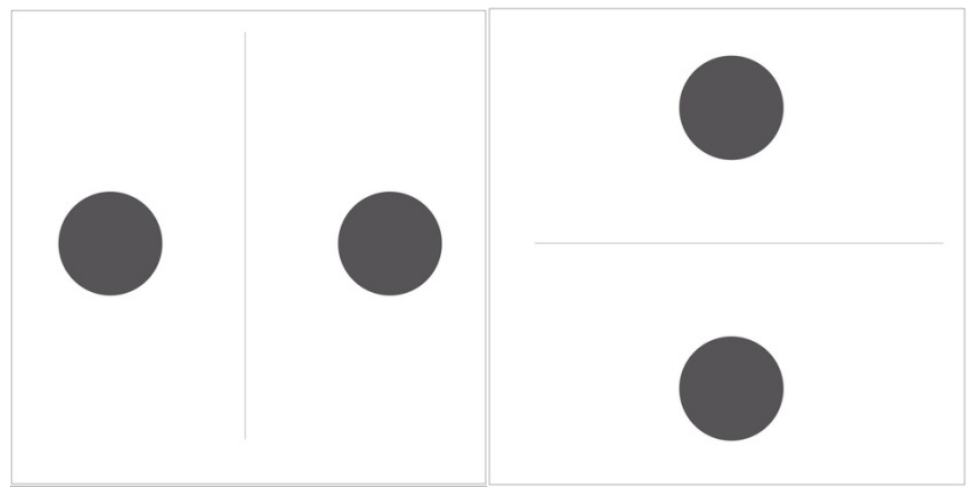

Vertical symmetry (left) Horizontal symmetry (right).

11 Balance Definition in Oxford Dictionary:

http://oxforddictionaries.com/definition/balance?region=us\&rskey=NT2ZE8\&result=1

12 Principles of Design http://char.txa.cornell.edu/language/principl/principl.htm 


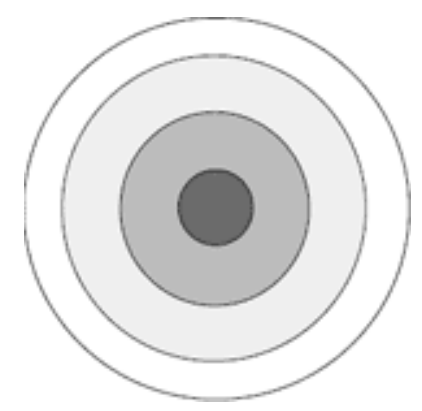

Radial symmetry

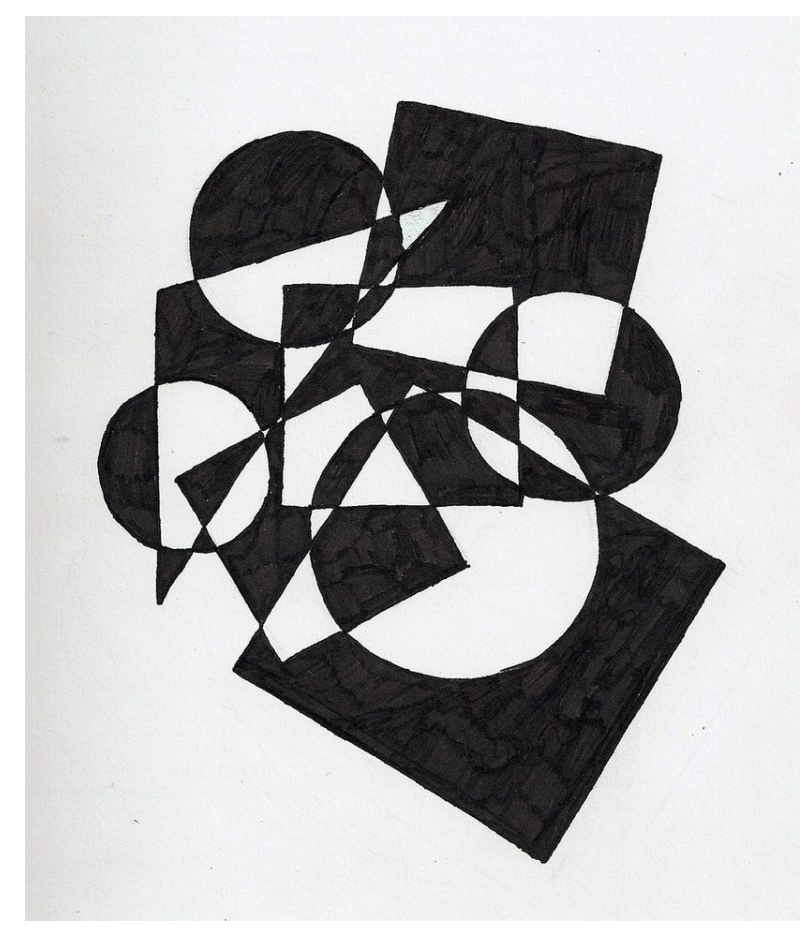

Asymmetrical balance $e^{13}$

Proportion refers to the relative size and scale of the various elements in a design, seeing the relationship between objects, or of parts of a whole. Proportion is discussed in terms of context or a standard. ${ }^{14}$

\footnotetext{
${ }^{13}$ Taken from: http://browse.deviantart.com/?q=asymmetrical\%20balance\&order=9\&offset=24\#/d2fuxi0

14 http://char.txa.cornell.edu/language/principl/principl.htm
} 


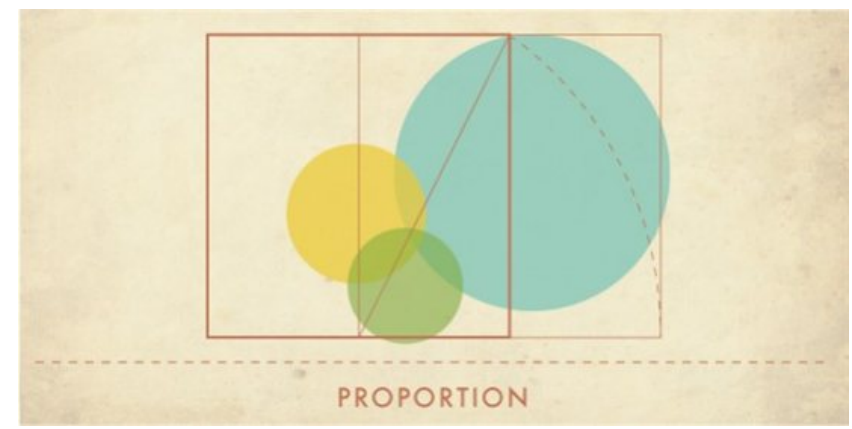

Proportion $^{15}$

In an analogue way to the notion of order, balance can presuppose:

- A set of things or people to be balanced.

- The proportion for achieving balance.

- The submission of the object to the proportion.

- The relations that derive from such submission to the balanced objects.

- The relation between the balanced and the balancing agent.

- The relation between the balanced and the proportion.

- The goal pursued by the balancing agent.

At the same time, the following principles can be stated:

- The object of balance in social system is external human behavior.

- The nature of the balance depends of the proportion used.

- Proportion can be conditioned by the nature of the objects, by the goals pursued by the balancer, and by the established order.

- Balance is instrumented by means of order.

Like with order, the conception and the implementation of balance comprises 3 aspects:

1. Conceiving the balance.

2. Electing the means to achieve the goal.

3. The effective accomplishment of the projected balance.

15 Image found here: http://blogs.office.com/b/office-show/archive/2010/06/09/design-principles-andanimation-in-powerpoint.aspx 
The interaction between order and balance can be explained with the following example:

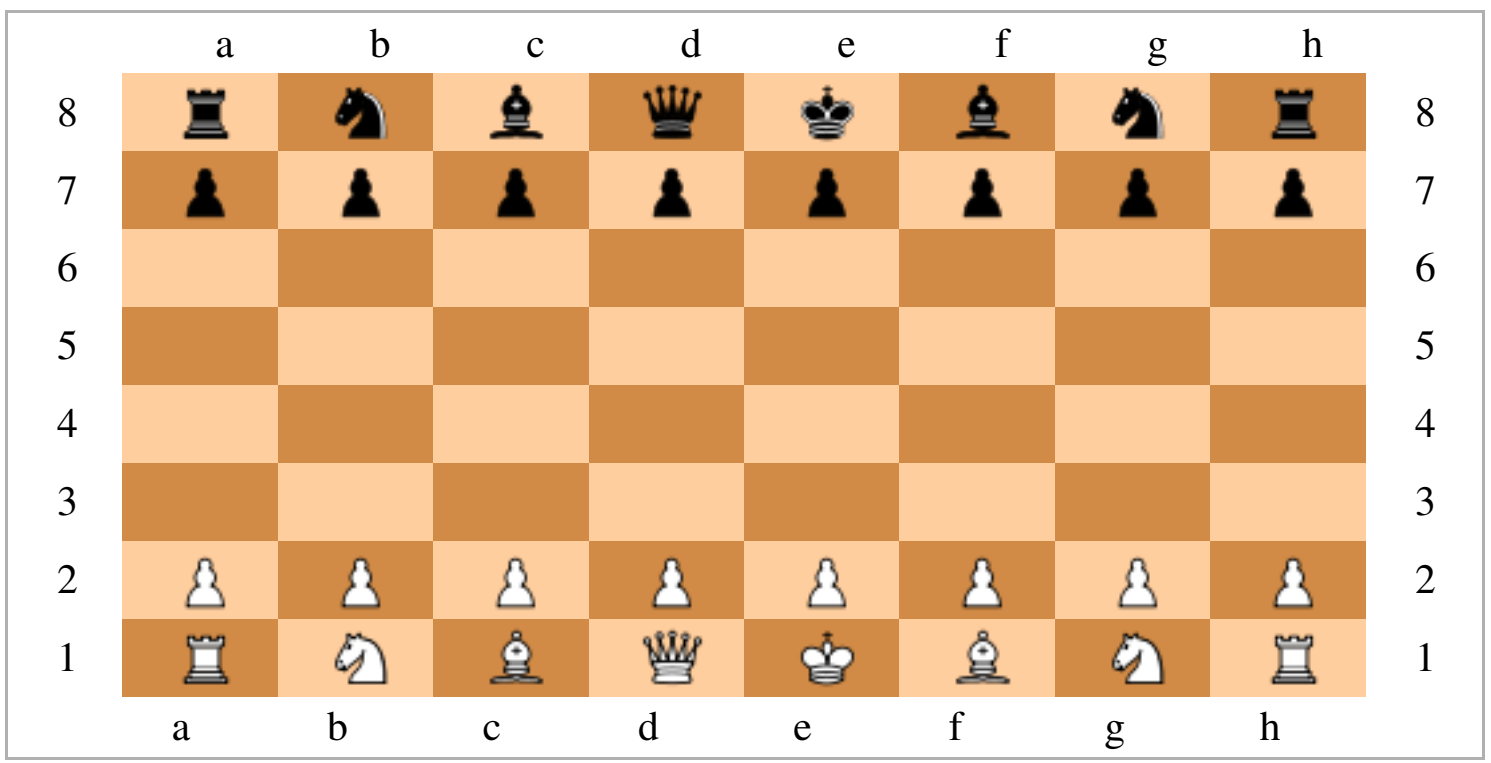

A standard chess configuration has both. It has a pattern that consists of 16 pieces per player aligned in 2 scores of 8 pieces and it has a proportion seeing that each player has the same amount of pieces arranged in a way that gives none of the opponents a starting advantage over the other. Now, suppose that the following arrangement is in place:

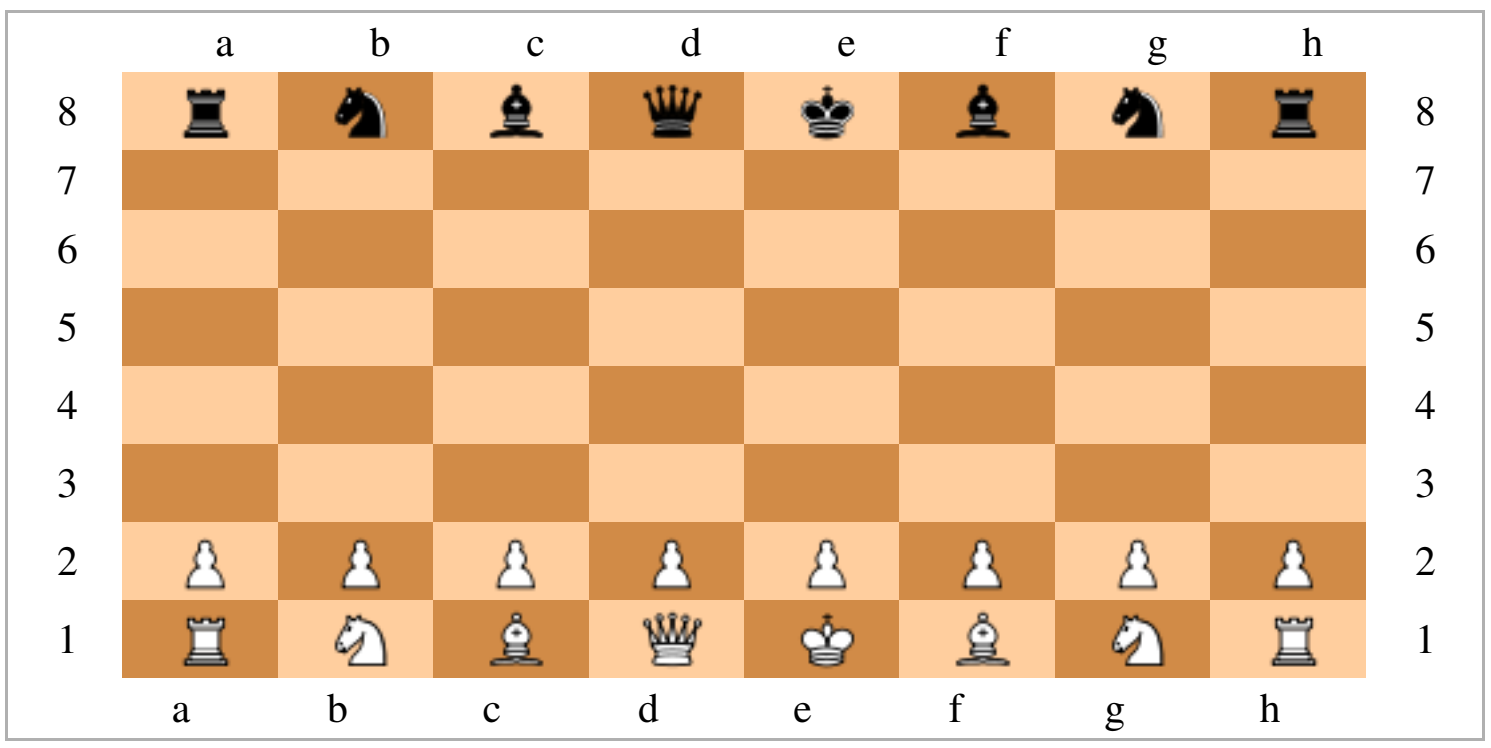




\section{Roberto Gustavo Mancilla}

In this case there is order but not balance (assuming the players have a similar level of skill), that is, there is a pattern but there is no proportion; there can be order without balance, but there cannot be balance without order, therefore, their relationship is asymmetrical. Now, the conjunction between order and balance brings forth the idea of Harmony; the latter can be defined as the conjunction between order and balance in a compatible relation; that is, it's the combination between a pattern and a proportion that although when coupled they put each other in a certain stress, they are able to function together.

To show that there can be multiple ways to join order and balance, the Chess 960 variant can be used as an example. Created by Bobby Fischer, it has the same pieces and boards that the standard chess game but it has new rules that allow the retaining of castling options and can result in 960 possible positions. The intention of this variant is to eliminate the advantage created by the memorization of opening moves, forcing players to rely on their talent and creativity. Here are two possible board positions given by the Chess960 variant:

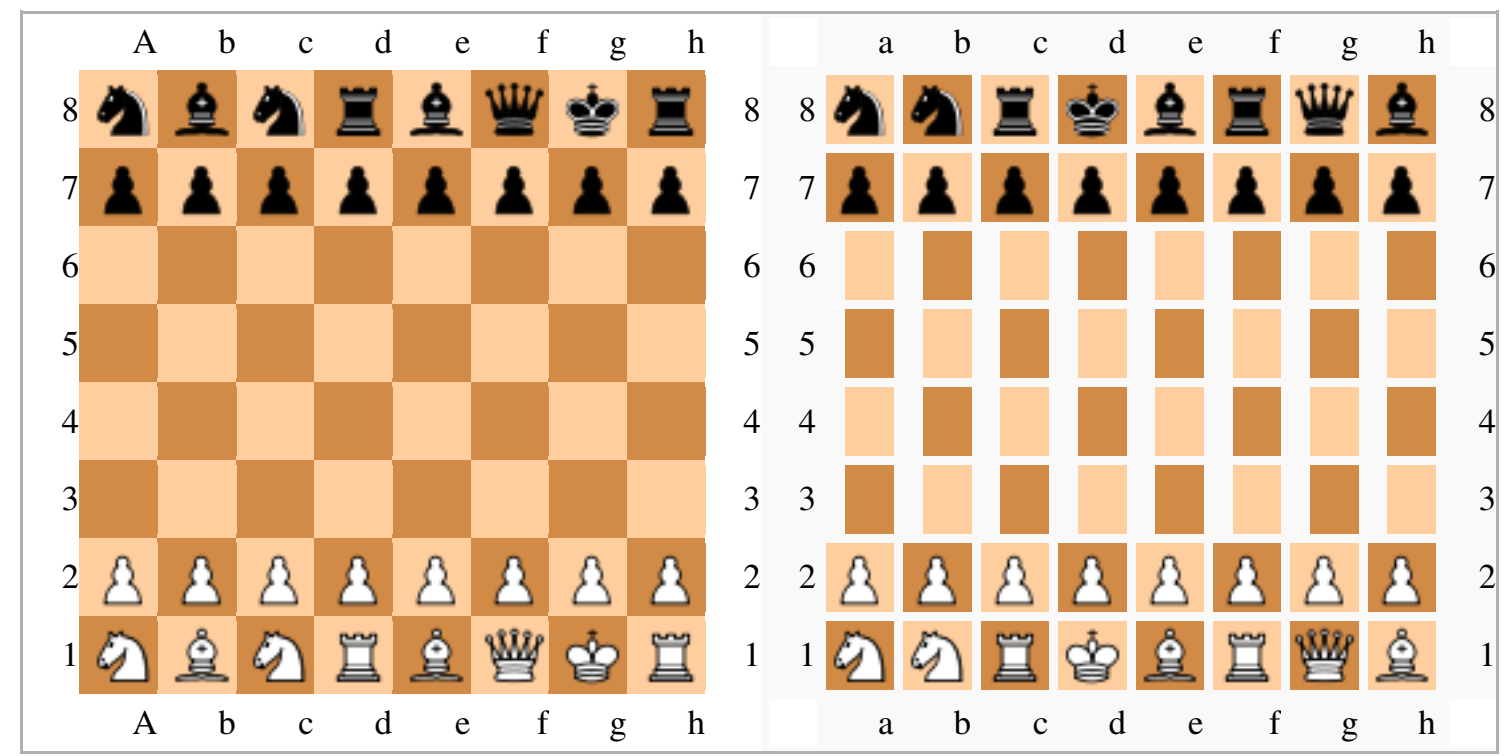

Both of these boards have compatible forms of order and balance (and thus harmony), indeed they have a pattern that orders the pieces of each of the players and a proportion that gives them equal chances of winning. 


\section{ii) Order, Balance and Harmony in Rationality}

The cognitive coherence is the goal of rationality as a thought machine and it is a form of harmony, as it consists of compatible forms of order and balance, and ideally should coexist and complement each other, although they are sometimes are at odds. Because order is the coping aspect of rationality, it can be said that it tends towards viability; which is a teleonomical concept because it tends towards adaptation and covers one of the two features of self observing systems, the other being teleology. Balance is teleological because the cognitive system can determine its purpose and goals beyond survival and can calibrate or recalibrate the latter by means of constant feedback with the environment; as order strives for viability, balance looks for optimality, which is the determination of goals and purposes, or what it considers best.

Both orderly and balanced rationality can be summarized from the process that Piaget (1974) describes as adaptation, upon which, any cognitive system regardless of its state of development establishes its needs and interests:

Every need tends to: $1^{\text {st }}$, to incorporate the things and people to the activity proper of the subject and, therefore, to "assimilate" the external world to the already built structures, and $2^{\text {nd }}$, to readjust the latter in accordance to the experienced transformations, and therefore, to "accommodate" them to the external objects... all mental life... tends to assimilate progressively the environment, and makes this incorporation thanks to a set of structures of psychic objects, which ratio of action is ever increasing: Perception and elemental movements first give access to proximate objects on its momentary state, then memory and practical intelligence allow to reconstruct its immediate anterior state and anticipate further transformations. Intuitive thought comes to reinforce both. Logical intelligence, in its form of concrete operations and finally of abstract deduction, ends this evolution by making the subject owner of the most distant happenings, both in space and time.

It is important to emphasize on the fact that the idea of order and balance is subject to the concept that the individual rational system has of it, as well of social and groupal rationality. Also, a rational system in the ideal sense has order and balance, that is, thought coherence has a pattern that secures viability and a proportion that seeks to optimize it. Rational systems can be such without having a clear idea of what constitute the goals of the system, that is, its balance. There can also be the case that the circumstances that surround such rational system does not allow the setting of a balance outside the pattern, because it can barely secure viability which can lead to balance being set on a short term basis. 


\section{iii) Constructivist epistemology. A brief introduction}

It was stated before that second order cybernetics deals with living systems and lower cognition and that fourth order cybernetics with the higher cognition and in general humans; a common thread between them is the biology of cognition, which takes on a constructivist approach. The latter is a theory of knowledge that centers on the active participation of the subject in building reality, instead of reflecting or representing it, rejecting the idea of an objective ontological reality perceived passively by means of the senses.

Maturana (2012) ${ }^{16}$ and also Varela (1998) ${ }^{17}$ espouse a constructivist theory of knowledge that has a biological basis, this has also been held by cyberneticists as von Foerster, von Glasersfeld, Pask and Ashby and also by psychologist Jean Piaget and his famous quote "The mind organizes the world by organizing itself". ${ }^{18}$ Martin Dougiamas, cited and summarized by Patsy Ann Johnson, makes the following classification of constructivist epistemology: ${ }^{19}$

- Trivial or Personal: Knowledge is actively constructed by the learner, not passively received from the environment.

- Radical: Coming to know is a process of dynamic adaptation towards a viable interpretations of experience. The knower does not discover truth about the real world.

- Social: Individuals participate in the learning of a collective, sometimes with what is learned distributed through the collective more than in the mind of one individual.

- Cultural: The ways in which individuals think are affected by the tools, artifacts, and symbolic systems used to facilitate social and cultural interaction.

- Critical: Myths that keep individuals from being empowered should be made visible and hence open to question.

\footnotetext{
${ }^{16}$ Maturana H.R., Varela F.J. (2012) Autopoiesis and Cognition. The Realization of the Living, D. Reidel Publishing Company, 79.

17 Maturana H.R., Varela F.J. (1998) The Tree of Knowledge. The Biological Roots of Human Understanding, Shambhala.

18 Ernst von Glasersfeld, Cybernetics, Experience and the Concept of Self, http://www.vonglasersfeld.com/056

19 Patsy Ann Johnson, Constructivism: A Short Summary, http://www.google.com.mx/url?sa=t\&rct=j\&q=\&esrc=s\&source=web\&cd=2\&ved=0CCAQFjAB\&url=ht tp $\% 3 \mathrm{~A} \% 2 \mathrm{~F} \% 2$ Facademics.sru.edu\%2Fcmste\%2FConstructivism4.doc\&ei=tLpbUMLXBKKYiQKBw4H ABw\&usg=AFQjCNELT4WLyhapguBAhZGhPjakZwJ6Bg\&kb=1
} 
Constructivism, as a theory of knowledge can be divided into two sets of theories: those that deal with knowing, and those that do so with learning. Before proceeding any further, it must be clarified that the human capacity of knowing is not a cognitive machine by itself, but the underlying feature of each and every one of them, that is, epistemic capacity is a requirement of any cognitive mechanism in order to be considered a cognitive machine. This is important to know because theories of knowing are centered towards the "order" aspect of cognitive coherence, which delve in the notion of viability, while theories of learning are drawn towards the "balance" aspect of cognitive coherence, because they can decide what are the goals to be set in learning and thus optimize.

Gordon Pask's (1975-6) constructive theory of learning known as conversation theory is a theory of learning, not of knowing, but it takes radical constructivism as a basis. von Foerster's phrase: "Anything said is said to an observer" leads from selfobserving systems to mutually observing systems, that is, social systems, which are the object of study of third order cybernetics.

This theory then, is a transition point between third and fourth order cybernetics, and foresees the interaction between rationality and language. He envisions learning from the context of a conversation, which is the basic unit of psychological and educational observation, with a strict conversation being the standard condition of conversation theory. This unit is comprised by the following elements: ${ }^{20}$

- The participants: Those who agree to the rules of a conversation language.

- Conversational domain: A representation of the topics in a subject matter.

- Epistemological commitment: A particular and canonical type of representation, which can be known or discussed.

- A topic: A subject to be understood.

- An understanding: The assimilation and learning of the topic within a strict conversation.

- Occasions: The intervals of time that precede reaching an understanding.

Pask (1975) distinguishes three levels of a conversation: Natural language (which comprises the level of a general discussion), object language (used in learning the topic) and Metalanguage (used for talking about learning). One striking feature of Paskian epistemology is that he creates a framework for understanding the participants and resources of the conversation in a way that precedes third and fourth order cybernetics

20 Pask G. (1976) Conversation Theory. Applications in Education and Epistemology, Elsevier, 3 and 4. 
by decades, but he centered towards first and second cybernetic aspects of learning, instead of attempting a cybernetics of human beings. 21

A conversation in the Paskian sense is a process of cognitive construction between two rational systems by means of a language (natural or otherwise) in which an understanding of a topic is achieved by means of continuous interaction that follows a circular causation process. Conversation theory leads to the conclusion that learning is a social activity and thus, much of knowledge is socially created and subject to groupal and social rationality. However, because Pask employs different subjects than those handled in fourth order cybernetics, only some general aspects of his theory will be adopted: Learning is made by the interaction between subjects and has a circular causational dynamic which help calibrate the assimilation/exchange of knowledge by means of repetition of interactions and clarification of used terms.

Adding on Pask (1975-6), Brier (2008) says that the biological capacity to construct knowledge is partly built into human perceptual systems, partly taught during childhood and reinforced by scientific training; the latter two presuppose culture and society. Humans as self-observing systems are product of the integration of their cognitive biological system and its ability to communicate socially with language. 22

Brier (2008) also says that human knowledge is the point of convergence between subjective and objective by means of the intersubjective in an ongoing and continuous process; the first being a cognitive system, the second a partly independent reality, and the third a language. He sets the following example:

When I see the apple tree in the garden, I do not create it as such; I merely create it in my world and give it social signification by fitting it into a recognized classification system. I do create an object in semiosis; if we do not realize this, we can easily fall into a solipsistic idealism.

To conclude this topic, a reflection must be made regarding substantive aspects of knowledge, that is, how it "feels", for much it has been said on how it is made. Interpreting Bergson, Barnard (2011) states that instead of visualizing our experiences as a dynamic flow, we tend to think of our inner experience as a linear series of static snapshots, this in part is due to the influence of language and logic.

The problem resides on the difference between language as a tool of cognition and the cognitive system that employs it. The former on the basis of differentiation and

21 Pask G. (1975) The Cybernetics of Human Learning and Performance. A Guide to Theory and Research, Hutchinson Educational, 13.

22 Brier S. (2008) Cybersemiotics, why information is not enough, University of Toronto Press, 211 and 229. 
sameness, that is, a word does not change its meaning; however, cognitive systems are constantly on flux:Words change of meaning, when an interpreter makes it so, that is, they are an observed system and they place this static constraints upon an observing system, which follows different dynamics. This means that although experiences and emotions are never the same they have to be adapted to a static and constant system of signs in order to express them. ${ }^{23}$

Brier (2008) helps explain this when he notes that because of the built-on processes of the animal stage, the human mind is semiotic in nature; so when speaking a language, those that use it are at the same time influenced by it for it is the framework upon which the cognitive system is built, or in his words: "Insofar we speak language, we are also spoken by language, which makes it difficult for us to think 'behind' language".

All this is important because this way of cognizing the world influences the way knowledge is constructed.

Bergson says that the way intelligence works is led by needs, thus it is relative; this is done by dividing things according to perspectives taken and then reconstructing them. This synthesis, while serving needs; it gives a general idea of things and not the thing itself. Bergson seeks to reverse this schema when he expounds his method of intuition. Rather than seeing the thing from the outside, intuition consists in entering into it, and this will lead absolute knowledge of the object of study; to Bergson, intuition is always about what is other. ${ }^{24}$

\section{iv) Social morphogenesis}

Morphogenesis can be understood as "the ensemble of mechanisms underlying the reproducible formation of patterns and structures and controlling their shape". 25 It functions under the premise that to understand a shape (morphology) it is necessary to understand its process of formation (morphogenesis), although the criteria for pattern formation and the function also influence this process. It studies inanimate and living shapes: They are differenced by the fact that external forces are the primary and direct cause of their shape of the former, while the latter is formed by internal forces although external forces can act as obstacles or constraints.

23 Barnard G.W. (2011) Living Consciousness. The Metaphysical Vision of Henri Bergson, SUNY Press, xxiii, $\mathrm{xxx}, 84,85$.

${ }^{24}$ Henri Bergson Definition Stanford Dictionary of Philosophy, , http://plato.stanford.edu/entries/bergson/

25 Bourgine P., Lesne A. (2006), Morphogenesis. Origins of Patterns and Shapes, Springer, 1. 
When it comes to cognition, morphogenesis can have two areas of study: In first place there is rational morphogenesis, which analyzes the shapes formed by human behavior in a reflexive context (self-observing system), then, social morphogenesis, studies the patterns formed by human behavior in an intersubjective context (mutually observing systems).

The shapes of the patterns produced by rational system can be studied in two fronts: those of cognitive coherence and those of rational epistemology, with both of them presenting aspects of order/pattern/viability/ teleonomy and balance/proportion/ optimality/ teleology. Social systems can be shaped by both internal and external forces, either acting separately or in conjunction with each other, that is, social morphogenesis is both teleological and teleonomical, because human beings can determine their own ends, be it individual or collectively, but at the same time they are shaped by the circumstances.

This process acts in three ways: Human behavior is shaped by one of both, by both are the same time or both teleology and teleonomy interact and influence each other to then operate upon human behavior. Behavior is shaped by internal forces (individual purpose) and external forces (physical environment and collective purposes). Cognitive morphogenesis (both social and rational) studies the "shapes" that human behavior can have; this has an intimate relation to the concepts of order and balance, and also with that of complexity.

Hierarchical dimension and fractal dimension are two important indicators of complexity, of which there is no agreement of what constitutes a proper parameter.Hierarchical dimension is the amount of subsystems that a system might have from the top to its most elementary subsystem. Society is complex because it is composed of many subsystems, which are product of several types of individual and collective interaction; a fractal is a self-similar geometric shape, and its dimension is the quantification of the number of copies that a object has at varying levels of magnification.

Society can be measured and studied by the hierarchies it possesses: a type of order for it is comprised by an object, a rule or set of rules, an orderer and his goals, a relation between object and rule, object to object and object to orderer. It also follows a process of conception of the order, selection of means and an implementation of it. Finally, human beings have a self-preservation pattern of cognitive coherence and a psychological disposition towards the creation and existence of a structure, that is, they seek certainty, or as Bergson envisioned it, they think in solids.

Balance is more related to self-similarity because all the ideas of social proportion through the implementation of values conceive society as a system of domination or 
their implementation has resulted in such. Domination has been a social paradigm of behavior that repeats itself through many configurations and theories, from Plato's Republic, in which Thrasymachus argues that it is the interest of the strong to Rawls and his Theory of Justice which envisions it as a form of liberalism.

The relationship between Order/hierarchy and Balance/self-similarity is one of implementation and dependence: the former is a vehicle for the latter, but both are creations of the human mind: They serve as ways to understand the world and as coping mechanisms against uncertainty or dissatisfaction of the existing structure; the human mind is configured to have expectations of certainty and at the same time, individuals have their own sense of order and balance, which coincide with the idea of authority legitimation and morality.

If Order tends to hierarchy and Balance to self-similarity, what is the morphogenetic structure that can be applied to Harmony? I propose that the answer is a mathematical phenomena known as universality. It was discovered in the 1950's in the energy spectrum of the uranium nucleus, it has also been noticed in the zeros of the Riemann zeta function, and oddly but not too surprising with someone acquainted with Mexican culture, in the Cuernavaca bus system. In a note made by Natalie Wolchover of Simons Science News, this phenomena is explained as follows: ${ }^{26}$

Each of these systems has a spectrum - a sequence like a bar code representing data such as energy levels, zeta zeros, bus departure times or signal speeds. In all the spectra, the same distinctive pattern appears: The data seem haphazardly distributed, and yet neighboring lines repel one another, lending a degree of regularity to their spacing. This fine balance between chaos and order, which is defined by a precise formula, also appears in a purely mathematical setting: It defines the spacing between the eigenvalues, or solutions, of a vast matrix filled with random numbers.

Universality is thought to arise when a system is very complex, consisting of many parts that strongly interact with each other to generate a spectrum. The pattern emerges in the spectrum of a random matrix, for example, because the matrix elements all enter into the calculation of that spectrum. But random matrices are merely "toy systems" that are of interest because they can be rigorously studied, while also being rich enough to model real-world systems, Vu said. Universality is much more widespread. Wigner's hypothesis (named after Eugene Wigner, the physicist who discovered universality in atomic spectra) asserts that all complex, correlated systems exhibit universality, from a crystal lattice to the internet.

\footnotetext{
26 Natalie Wolchover, In Mysterious Pattern, Math and Nature Converge, WIRED MAGAZINE, February 6, 2013. http://www.wired.com/wiredscience/2013/02/math-and-nature-universality/all/
} 
It appears then that this idea might be applicable to Harmony, as it has been found in social systems, quantum physics and living systems; this idea can be summarized as what is commonly known as "ordered disorder" or "functional disorder"; an apparent chaos that at the same time has a degree of regularity. This can be exemplified as follows:

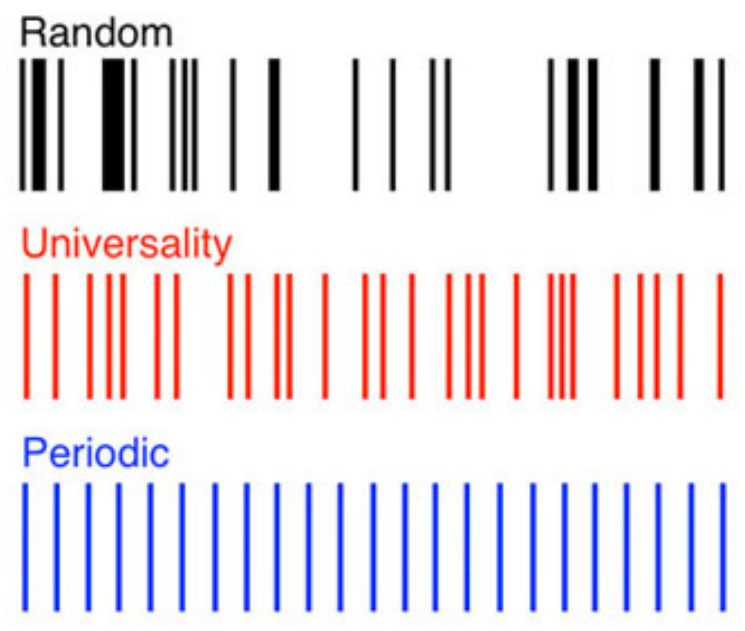

Finally, an idea of cognitive morphogenesis is advanced; it is the study of the forms of human behavior and relates to the idea of order, balance and harmony by coupling them with complexity: hierarchical dimension, fractal dimension and universality. The thing all these concepts have in common is that they are the result of repetition, that is, they depend on the continuous performance of certain behaviors, in the same ways that machines require use to acquire meaning or living systems the repetition of cycles to endure. The repetition of behavior is then measure of complexity on rational and social systems.

\section{The relationship between third and fourth order cybernetics}

\section{a) Rationality and Language}

Once that both language and rationality have been explained as omnipoietic cognitive machines, it is most useful to see the relationship that they hold, in which they act as complement. Piaget's typology of cognitive development is useful in the analysis, his 
comments on the relationship between language and intelligence on the sensorimotor stage prove to be of interest:

Intelligence, by the way, appears much before than language, that is, much more than the internal thought that supposes the usage of verbal signs (of the interiorized language). But it is only an exclusively practical intelligence, that is applied to the manipulation of objects and does not use, instead of words and concepts, but perceptions and movements organized in schemas for action.

Though coherence appears in this stage not as an exercise of reflection, but as a practical schema of action built by interaction and experience of sensory and motor reflexes. This changes completely with the incorporation of language at the outset of the pre-operational stage:

With the appearance of language, the conducts result deeply affected, both in their affective and intellectual aspects... the child acquires, thanks to language, the capacity to reconstruct his prior actions in the form of a story and to anticipate his future actions by means of verbal representation. This has three essential consequences for mental development: a possible exchange between individuals, that is, the start of socialization; an interiorizing of words, that is, the appearance of thought properly stated, that has as a basis the inner language and the system of signs, and above all, an interiorization of action as such...

Of this it can be concluded that rationality cannot be possible without language, otherwise, it would have stayed within lower cognition as a form of practical reasoning. That is, language is what creates the transition from lower condition, found in second order cybernetics, to that of third and fourth order. This author summarizes it in the following phrase:

When the appearance of language intervenes, the child is confronted, not only with a physical universe like before, but with two new worlds... the social world and the world of internal representations.

Another point in the relationship between language and reason is that rationality in part creates and recreates itself by means of a social process, which is fostered in part by the existence of language, which acts as a bridge between cognitions. However, language also depends on rationality, because if it were not of cognitive coherence as a maintainer of the cognitive system in general, language could not be exercised because there would not be a system left to do it. This means that although language is the basis of rationality, without the latter, the former could not subsist.

The relationship between language and reason (both of which are part of high cognition) can be seen more clearly, when it is related to the cognitive system in 
general. Language is set as a cognitive machine that allows the usage of symbols and inner representation, on the other hand, rationality takes that cognitive input and output and that of other machines and integrates them into a whole that can be restructured as new information comes along.

\section{b) Application of fourth order cybernetics to Society, Power, Culture and Institutions}

It was seen on the prior papers the way in which language as a cognitive machine can be applied to Society, Power, Culture and Institutions; rationality complements their treatment by providing a useful framework for understanding individual decision making vis a vis a social context and also to understand the interactions between individuals and their environment. When high cognitive systems relate to one another by means of language and create institutional facts to serve a specific function, they create different groups with different functions that interact and overlap between them; rationality as thought coherence configured in its different levels, (individual, groupal and social) is a point of convergence between third and fourth order cybernetics, that is, it is a way in which individual and social systems interact.

This is represented in the many ways in which power is exercised: with traditional notions as the manipulation of preferences and as the social context. Institutions are the ways in which the usage of Power can be used within a social context within a specific pattern and proportion, however, rationality in all its levels manifest itself to both those who apply the existing rules either in voluntary or compulsory ways and those who receive the policy contained within them which is applies to their circumstances. Notions of Justice, or social proportion, are part of the individual level of rationality, for they determine how the individual must behave, and at the same time are part of the groupal and social notions, because it acts as the proportion of the existing hierarchies of Power.

\section{c) Sociocybernetics}

Sociocybernetics has been understood as the application of first and second order cybernetics and systems theory to social systems. However, it has been shown in these papers that these are insufficient and that the aim of sociocybernetics should be the creation of a specific paradigm, in this case that of cognitive machines, instead of extending autopoiesis from biology to social systems.

Sociocybernetics can then be understood as the interplay between third and fourth order cybernetics for the purpose of understanding human behavior in an individual and collective scale, with first and second cybernetics functioning as complements. The 
main field of application of sociocybernetics is the interplay between the different levels of rationality, but it can also extend to the study of language and speech acts as creators of institutional facts and to thought coherence as a basis of means ends analysis under a specific set of social contexts.

\section{Conclusions}

FIRST.- Self-consciousness is the point of transition between lower and human cognition. The latter can be understood as the processing of information made by an autopoietic system in its interaction with its surroundings with the possibility of stating a purpose beyond self-sustainment.

SECOND.- Fourth order cybernetics deals with the study of self-observing systems, which are both teleological and teleonomical; it studies cognitive machines, information processing mechanisms of the high order that have their basis within the neural network of human beings. The one which is studied preeminently is rationality, which is a mechanism which allows the development of coherence within the thought system.

THIRD.- Rationality can be individual, groupal and social. They can interact and be at odds with each other, when the latter happens there is a cognitive dissonance.

FOURTH.- Hermeneutics from a cybernetic perspective can be seen from the perspective of patterns (order), proportions (balance) and the functional implementation of both (harmony). This can be applied in the realm of third and fourth order cybernetics, and they are relative perspectives in nature, since they originate in an observer.

FIFTH.- Cognitive coherence encompasses both an aspect of order (pattern establishment/viability of the system/teleonomy) and of balance ( proportion of the pattern/ optimality of the system/ teleology).

SIXTH.- Constructive epistemology states that knowledge is not passively received, but actively constructed. It encompasses knowing and learning, which both comprise also the order and balance aspects of thought coherence

SEVENTH.- Cognitive morphogenesis is the study of how forms of human behavior originate; it can be applied to third and fourth order cybernetics. In social systems, order/pattern/viability can be related to hierarchies, balance/proportions/optimality can be related to self-similarity and their functional implementation to universality.

EIGHT.- Rationality and Languages are complements, the former is developed by the capacity of symbol creation and abstraction, but the latter could not subsist without 
thought coherence. Rationality can be applied to Society, Culture and Institutions by means of the usage of levels of rationality to constitute models of decision making.

NINTH..- Sociocybernetics can be defined as the interplay between third and fourth order cybernetics for the purpose of understanding human behavior in an individual and collective scale, with first and second cybernetics functioning as complements.

\section{Sources}

\section{Electronic}

Ernst von Glasersfeld, Cybernetics, Experience and the Concept of Self, Retrieved from http://www.vonglasersfeld.com/056

Ernst von Glasersfeld, An Exposition of Constructivism: Why Some Like it Radical, Retrieved from http://www.oikos.org/constructivism.htm

Patsy Ann Johnson, Constructivism: A Short Summary, Retrieved from http://www.google.com.mx/url?sa=t\&rct=j\&q=\&esrc=s\&source=web\&cd=2\&ved=0CCA QFjAB\&url=http $\% 3 \mathrm{~A} \% 2 \mathrm{~F} \% 2 \mathrm{Facademics}$. sru.edu\%2Fcmste\%2FConstructivism4.doc\&ei $=$ tLpbUMLXBKKYiQKBw4HABw\&usg=AFQjCNELT4WLyhapguBAhZGhPjakZwJ6Bg $\& \mathrm{~kb}=1$

Oxford Dictionary http://oxforddictionaries.com/

Stanford Dictionary of Philosophy, http://plato.stanford.edu/

Natalie Wolchover, In Mysterious Pattern, Math and Nature Converge, WIRED MAGAZINE, February 6, 2013. Retrieved from http://www.wired.com/wiredscience/2013/02/math-andnature-universality/all/

\section{Bibliographical}

Ashby W. R. (1965) Introduction to Cybernetics, Chapman Hall Ltd.

Barnard G.W. (2011) Living Consciousness. The Metaphysical Vision of Henri Bergson, SUNY Press.

Bourgine P., Lesne A. (2006), Morphogenesis. Origins of Patterns and Shapes, Springer.

Brier S. (2008) Cybersemiotics, why information is not enough, University of Toronto Press.

Maturana H.R., Varela F.J. (1998) The Tree of Knowledge. The Biological Roots of Human Understanding, Shambhala.

Maturana H.R., Varela F.J. (2012) Autopoiesis and Cognition. The Realization of the Living, D. Reidel Publishing Company.

Máynez E.G. (1974) Filosofía del Derecho[Legal Philosophy], Porrúa.

Nakhnikian G.(1954), A Note on W. Donald Oliver's Theory of Order, PHILOSOPHY OF SCIENCE vol. 21, No. 2. 
Introduction to Sociocybernetics, Part 3: Fourth Order Cybernetics

Oliver W. D. (1951) Theory of Order, The Antioch Press.

Pask G. (1976) Conversation Theory. Applications in Education and Epistemology, Elsevier.

Pask G. (1975) The Cybernetics of Human Learning and Performance. A Guide to Theory and Research, Hutchinson Educational.

Piaget J., Petit N. (trans.) (1974) Seis Estudios de Psicologia[Six Studies in Psychology], Editorial Seix Barral.

Stillings N.A., Weisler S.E., Chase C., Feinstein M.H., Garfield J.L., Rissland E.L. (1995) Cognitive Science. An Introduction, (2nd ed.), MIT Press.

Zangeneh M., Haydon E. (2004), The Psycho-Structural Cybernetic Model, Feedback, and Problem Gambling: A New Theoretical Approach, InTERnAtional Journal of Mental Health AND Addiction, Vol. 1, No. 2. 